\section{PWE-017 PRODROMAL IRRITABLE BOWEL SYNDROME DOUBLES DISEASE LAT ENCY LENGTH IN CROHN'S DISEASE AND INCREASES IT BY A THIRD IN COELIAC DISEASE: NATURAL HISTORY OR TUNNEL VISION?}

doi:10.1136/gut.2011.239301.280

S M Barratt, * J S Leeds, K Robinson, A J Lobo, M E Mcalindon, D S Sanders The GI \& Liver Unit, Sheffield Teaching Hospitals, Sheffield, UK

Introduction Previous studies report that patients with Coeliac Disease and Inflammatory Bowel Disease (IBD) (Crohn's Disease (CD) and Ulcerative Colitis (UC)) experience a latency period (a period between the onset of symptoms and correct diagnosis). Irritable Bowel Syndrome (IBS) is more prevalent in Coeliac Disease and IBD. We aimed to determine the prevalence of prodromal IBS (P-IBS) during such latency periods and the impact P-IBS has, if any, on latency period duration.

Methods 683 histologically proven age and sex matched patients (Coeliac $n=225,26 \%$ male, mean disease duration 8 years; $\mathrm{UC} n=228,43 \%$ male, mean disease duration 16.5 years; $\mathrm{CD} n=230,28 \%$ male, mean disease duration 15.5 years) completed a postal survey. Patients were asked to recall if they perceived a 'latency period' and whether they had been diagnosed with IBS by a physician during this period. Responses were validated by medical records.

Results 100/225 (44\%) of Coeliac patients reported a latency (mean length 13.2 years.) Of these 100 patients 67 (67\%) had P-IBS. Mean latency length in P-IBS Coeliac Disease was 10 years versus 7 years without $(p=0.046)$. In IBD overall $326 / 458(71 \%)$ reported a latency (mean length 4.99 years) of which $106(32 \%)$ had P- IBS. Mean latency length in P-IBS IBD was 3 years versus 1.5 years without $(p=0.01)$. In UC $110 / 228(48 \%)$ reported a latency (mean length 2.89 years) of which $42(38 \%)$ had P-IBS. There was no difference in mean latency length in P-IBS UC versus those without, both 1 year in length ( $p=n s)$. In CD 216/230 (94\%) reported a latency (mean length 6.15 years) of which 64 (29\%) had P-IBS CD. Mean latency length in P-IBS CD was 4 years versus 2 years without $(p=0.018)$.

Conclusion When present, the duration of latency in patients with Coeliac Disease is significantly longer and more often characterised by P-IBS than patients with IBD. In both Coeliac Disease and CD P-IBS increases the duration of the latency period, significantly so in CD. This delay may (1) reflect inherent diagnostic difficulties of symptoms attributed to small bowel pathology (2) suggest that IBS forms part of the natural history of Coeliac Disease and CD (3) demonstrate that once a diagnosis of IBS is reached physicians are more likely to attribute ongoing symptoms to refractory IBS and not to an underlying small bowel inflammatory process, such as Coeliac Disease or IBD.

Competing interests None.

Keywords coeliac disease, IBS, inflammatory bowel disease, prodrome. 